\title{
LE \\ PROBLÈME DES PORTES DE FER
}

\author{
PAR \\ B. KNEŽEVIĆ *
}

On désigne sous le nom de Portes de Fer le défilé du Danube entre les ruines de l'ancienne forteresse du Golubac sur la rive yougoslave et la ville roumaine de Turnu Severin située bien plus en aval sur la rive gauche du Danube. Ce secteur du fleuve, long de plus de $100 \mathrm{lkm}$, doit son nom de Portes de Fer aux cataractes sur les roches du lit élargi, entre le village yougoslave de Sip et le village roumain de Gura Vaii. Toute une nuée de rochers jaillit ici au-dessus des basses eaux sur une longueur de plus de $2 \mathrm{~km}$.

Sur une longueur de $117 \mathrm{~km}$, du $\mathrm{km} 931$ an km 1048 - comptant de l'embouchure du bras de Sulina dans la mer Noire - il y a cind cataractes semblables et c'est avec raison que tout le défilé a recu le nom des premières cataractes dans le passage vers l'amont. De toute la différence de niveau, aux eaux moyennes, plus de $26 \mathrm{~m}$ du commencement à la fin du défilé, $20,8 \mathrm{~m}$ sont concentrés aux cinq endroits cités ci-dessous, en descendant vers l'aval :

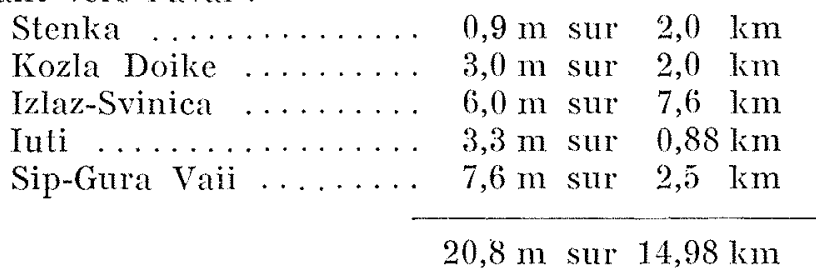

Le reste de la chute, environ $5,5 \mathrm{~m}$, est réparti sur les autres parties du secteur des Portes de Fer sur une longueur de $102 \mathrm{~km}$, où souvent la vitesse moyeme du profil ne dépasse pas $50 \mathrm{~cm} / \mathrm{s}$.

Les Portes de Fer ne représentent pas un défilé unique, mais bien quatre défilés séparés par trois vallons. La largeur du fleuve varie en conséquence, atteignant jusqu'à $2000 \mathrm{~m}$, pour se réduire dans les parties étroites à 300,200 et même $150 \mathrm{~m}$.

* Ancien professear à la Faculté de Génie civil, Belgrade.
Dans les parties élroites du défilé, telles que le Gospodjin Vir, le Danube coupe les plus hautes chaînes des Karpates méridionales, où les berges abruptes s'élancent à plus de $300 \mathrm{~m}$ au-dessus de l'eau.

Les vieux bateliers racontent que dans une poche profonde du défilé, près du seuil Piatra Lunga, un chaland coulé est resté debout et que l'eau au-dessus de Iui était profonde de plus de $10 \mathrm{~m}$. Dans un autre vortex du groupe Gospodjin Vir, on pouvait très bien entendre le bruit des cailloux polissant les parois du profond entonnoir [1]. Dans ce même remous, on prenait auparavant, jusqu'au commencement des travaux de régularisation dans la dernière décennie du $\mathrm{x}_{\mathrm{I}} \mathrm{x}^{*}$ siècle, des esturgeons pesant à peu près $800 \mathrm{~kg}$, qui remontaient des eaux calmes du Delta jusqu'aux Portes de Fer [2].

Dans les Grands Cazanes, le Danube a des berges très hautes et presque verticales; son lit est large de $150 \mathrm{~m}$ et par endroits profond de plus de $50 \mathrm{~m}$, de sorte que son fond s'abaisse au-dessous du niveau de la mer, éloignée de plus de $1000 \mathrm{~km}$. L'énorme masse d'eau en mouvement, les barrièes rocheuses fréquentes dans le lit, les variations de la vitesse du courant de $0, \bar{a}$ à $5 \mathrm{~m} / \mathrm{s}$, la longueur du défilé de plus de $100 \mathrm{~km}$ et les blocs terrifiants des montagnes, dressés au-dessus du fleuve, font de ce défilé une cuvre majestueuse de la nature.

Dès les temps les plus anciens, on a tenté d'utiliser le fleuve pour la navigation dans toute la longueur du défilé; le percement des routes el leur entretien à travers les hauteurs boisées et inhospitalières n'étant pas moins difficiles et périlleux. Un vestige de ces efforts du temps de la domination romaine dans ces contrées est visible dans l'inscription gravée dans le rocher, et connue sous le nom de Tabula Traiana ( $\mathrm{km} \mathrm{967),} \mathrm{à} \mathrm{côté} \mathrm{de} \mathrm{laquelle}$ passait le chemin taillé dans le rocher et supporté par des consoles, sur lequel des chevaux halaient autrefois de petites embarcations vers l'amont. 


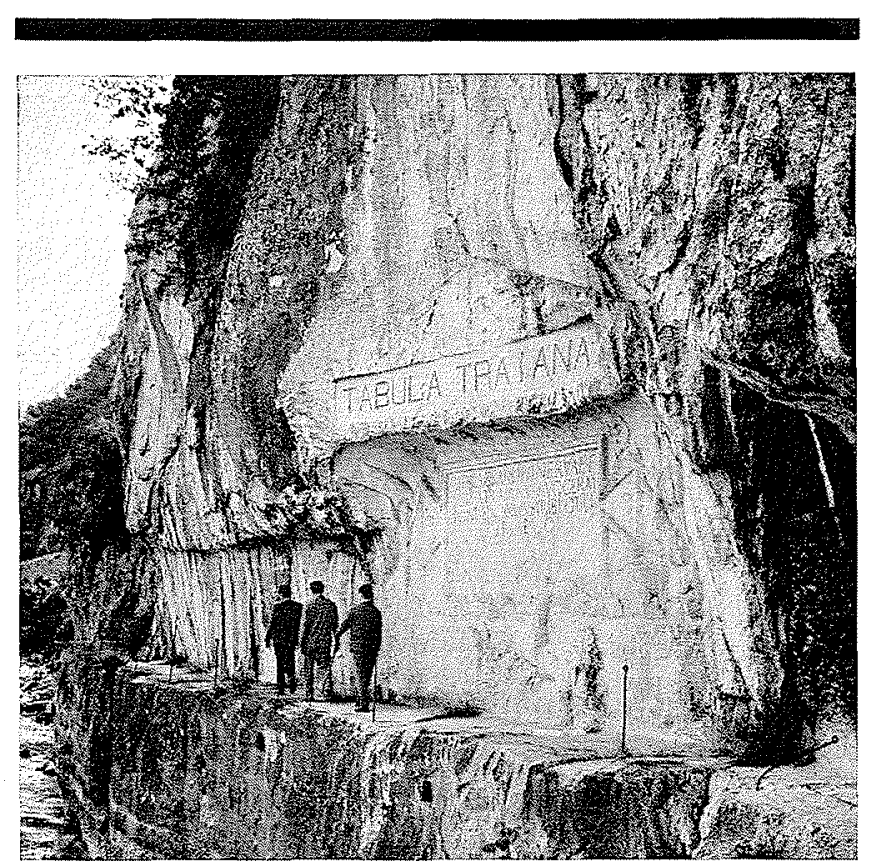

Tabula Trajana, sur la rive droite.

Tabula Trajana am rechten Vjer.

Cet état de choses s'est maintenu pendant des siècles et ce n'est que vers le commencement du $\mathrm{XrX}^{\prime}$ siècle qu'avec l'apparition de la traction à vapeur et le changement des conditions politiques dans la Péninsule balkanique, que le Danube des Portes de Fer est devenu l'objet de sérieuses études sur les moyens d'enlèvement des obstacles à la navigation dans son lit rocheux.

\section{Travaux \\ de régularisation du secteur en vue de la navigation}

Les premiers levés pour l'établissement des projets et les premier's projets sont l'œuvre de l'ingénieur hongrois Pal Vásárhelyi, de 1832 à 1834. Ses projets prévoyaient la régularisation du secteur par le creusement de chenaux dans les parties rocheuses et peu profondes du lit. Vâsárhelyi a entrepris en 1834 de petits travaux de dérochage du lit lors de très bas niveaux d'eau. Bientôt après, le premier batcau à vapeur, avec le nom symbolique d' «Argo», a traversé les Portes de Fer. Il avait une machine de $52 \mathrm{ch}$ et une jauge de 242 tonnes.

Pareils à ceux de Vásárhelyi furent d'autres projets et propositions que l'on soumettait successivement à l'examen et aux décisions. Tous ces projets ont été examinés par une commission composée d'experts étrangers. Cette commission a présenté ses propres propositions qui différaient un peu des propositions antérieures; et, pour la traversée des cataractes de Sip, elle proposait comme aussi l'ingénieur américain MacAlpine en 1874 - un canal à écluses.

Le Conseil technique hongrois a accepté ces propositions en 1833, excepté celles concernant la section Djerdap-Sip, où il fut décidé de construire un canal à courant libre sur la rive droite du fleuve. Ainsi, cinquante ans après les premières délibérations techniques concernant la navigation à travers le défilé des Portes de $\mathrm{Fer}$, on était arrivé à la solution d'après laquelle la Direction technique hongroise commença six ans plus tard les travaux de régularisation qui allaient être tous achevés en 1900. Le secteur a été divisé en deux sections : la section amont, du $\mathrm{km} 1048$ au $\mathrm{km} 952$ avec une largeur de $60 \mathrm{~m}$ pour les chenaux dans les cataractes et avec la profondeur de $2 \mathrm{~m}$ au niveau correspondant au zéro de l'échelle à Orsova; la section aval, du $\mathrm{km} 952$ au $\mathrm{km} 931$, pour laquelle fut fixée une profondeur de $3 \mathrm{~m}$ et une largeur de $60 \mathrm{~m}$, sauf dans le canal de Sip, dont la largeur fut portée à $70 \mathrm{~m}$ de facon à rendre possible le croisement des bateaux.

Le schéma, projeté et exécuté, de la régularisation est très simple et se réduit au creusement de chenaux à travers les cataractes à la profondeur nécessaire à la navigation aux basses eaux. Si le lit, en aval du chenal creusé, présente une largeur trop grande, on élève dans le prolongement des chenaux des digues longitudinales qui répartissent la différence du niveau de l'eau sur toute la longueur de la voie navigable.

Des difficultés particulières pour la réalisation d'une voie navigable sûre se manifestaient surtout aux cataractes de Iuti et de Sip. A cette derniere, la voie navigable est creusée à côté de la rive droite et sur une longueur de $1834 \mathrm{~m}$. Le canal est séparé du lit du flenve par une digue en gros bloes de pierres, s'élevant au-dessus des plus hautes eaux observées. La grande vitesse du courant dans le canal eut pour conséquence l'établissement d'une traction auxiliaire qui, de 1900 à 1933, fut assurée par un toueur et, depuis 1933 à nos jours, par la traction sur berges au moyen de locomotives de chemin de fer. L'introduction de cette traction a grandement amélioré les conditions de la navigation dans le canal de Sip et par conséquent de la navigation entière dans le Danube moyen.

Au cours de vingt années de travaux en vue de l'aménagement des cataractes pour la navigation, on a creusé sous l'eau $15470 \mathrm{~m}$ de chenaux, en extrayant plus d'un million de $\mathrm{m}^{3}$ de matériaux. On a en outre construit $19 \mathrm{~km}$ de digues longitudinales, dans lesquelles on a mis 1,2 million de $\mathrm{m}^{3}$ de pierres. Pour tous les travaux et la construction du quai au port d'Orsova, on a dépensé 33300000 couronnes austro-hongroises. Pour les travaux supplémentaires et les frais de matériel et de personnel, le Service de la navigation du Bas Danube a dépensé, de 1899 à 1914, la somme de 4933000 couronnes, en réalisant pour la même période une recette de 12820000 couronnes.

Il est hors de doute que tous ces travaux, ainsi que les travaux complémentaires faits plus tard, parmi lesquels le plus important est l'établissement de la traction sur berge a Sip, ont rendu possible la navigation et ont grandement contribué à son développement. Cependant, de nombreuses difficultés ont subsisté : ceci diminue la valeur des Portes de Fer comme voie de grande navigation fluviale que l'on peut prévoir sur le Danube. Il suffit d'en citer trois: la circulation des bateaux à voie unique dans les passes étroites et les chenaux creusés, l'interdiction de la navigation de nuit et l'impossibilité d'utiliser la pleine charge des bateaux aux basses eaux.

A cause de la largeur réduite des chenaux creusés et des grandes vitesses de courant prévues dans ceux-ci, on a dû apporter un grand soin au balisage de la voie navigable et au service de signalisation tout le long du secteur; ceci d'autant plus que, dans les parties non régularisées du fleuve, il était 
resté quelques endroits difficiles pour le libre passage des bateaux. On a établi dans le premier temps sept, et après 1919, encore quatre stations de signalisation, qui, au moyen de ballons et de pavillons, indiquent aux bâtiments si le passage dans les parties étroites de la voie navigable est libre. Elles sont reliées par des lignes téléphoniques. Puisque, sauf au canal de Sip, les bords de la voie navigable creusée ne sont pas visibles, on a placé sur ces bords cinquante-quatre signaux flottants, qui, par leur forme et leur couleur, indiquent les deux côtés de la voie de navigation et montrent la direction du courant aux endroits marqués. Enfin, chaque bateau reçoit à bord un pilote spécialement formé et expérimenté qui conduit le bateau dans tout le secteur des Portes de Fer.

\section{Utilisation du potentiel hydraulique}

Au cours du $x^{\circ} x^{\circ}$ et au commencement du $\mathrm{xx}^{\circ}$ siècles, les circonstances et les conditions politiques générales et économiques ont permis la régularisation du Danube aux Portes de Fer pour la navigation, mais elles n'étaient pas favorables à une utilisation énergétique du fleuve. De nos jours, dans la deuxième moitié $\mathrm{d} u \mathrm{xx}^{\circ}$ siècle, ces conditions existent et les deux pays riverains, la Roumanie et la Yougoslavie, abordent d'un commun accord et avec décision l'étude du problème des forces hydrauliques des Portes de Fer et d'une amélioration plus poussée des conditions de navigation dans ce secteur. L'administration mixte, roumaine-yougoslave, du secteur est en train d'achever les projets et de faire des préparatifs pour la construction d'une des plus importantes centrales hydrauliques d'Europe.

Surmonter les obstacles à la navigation vers la fin du $x x^{*}$ siecle signifiait une lutte contre les qualités défavorables des Portes de Fer; l'initiative nouvelle représente la lutte pour ce qui est le meilleur aux Portes de Fer, le potentiel énergétique. Tous les ouvrages de la future usine gigantesque et des installations de navigation, ainsi que le futur bassin d'accumulation, se trouvent sur les territoires de la Yougoslavie et de la Roumanie, c'està-dire entre leurs frontières sur le Danube. Les pays voisins de l'Europe sud-orientale portent également un grand intérêt à l'énergie électrique aux Portes de Fer, et surtout à l'amélioration de la navigation. L'énergie _ plus de $10,5.10^{9} \mathrm{kWh}$ que fournissent les Portes de Fer peut augmenter considérablement le capital énergétique des deux pays, Roumanie et Yougoslavie, et améliorer l'exploitation de leurs systèmes énergétiques.

Tout ceci, ainsi qu'une série d'autres avantages et utilités que les Portes de Fer présentent, pousse les deux pays à régler en commun tous les problèmes que pose la construction d'un ouvrage technique aussi grand qu'un barrage avec usines et écluses de navigation sur le deuxième fleuve de l'Europe.

\section{Les possibilités techniques}

Il faut noter tout d'abord que, sur le secteur limitrophe roumain-yougoslave, il existe des possibilités d'utilisation énergétique, non seulement dans le secteur des Portes de Fer, mais aussi en aval de celui-ci. Du potentiel total utilisable, plus de $80 \%$ se trouvent dans le secteur des Portes de
Fer et moins de $20 \%$ dans le secteur aval. Le secteur entier ne peut pourtant ètre aménagé en une seule chute, du fait de la faible altitude des rives et des terrains avoisinants en aval des Portes de Fer, et par suite deux chutes semblent indiquées. L'une pourrait se situer au $\mathrm{km} 852$ (Gruia) alors que l'autre pourrait être placée au profil SipGura Vaii (km 943) ou bien à Orsova ou Orgadina ( $\mathrm{km} \mathrm{955,962)} \mathrm{ou} \mathrm{enfin} \mathrm{à} \mathrm{Iuti} \mathrm{(km} \mathrm{988).} \mathrm{Les} \mathrm{profils}$ Orsova, Ogradina et Iuti se trouvent en amont de la grande cataracte près de Sip, de sorte que la perte de chute de $7,6 \mathrm{~m}$ à ces endroits pourrait être compensée par le rehaussement du niveau normal à l'usine aval de Gruia jusqu'à la cote 48, ce qui ne serait pas commode, comme il a été dit, à cause des vastes terrains riverains à faible altitude. Pour cela, la meilleure solution pour l'aménagement du secteur limitrophe entier parait celle à deux chutes, celle de Sip-Gura Vaii dans la première étape, et celle de Gruia, avec une cote de retenue réduite, dans la deuxiène étape de construction.

On a également étudié l'aménagement du Danube roumain-yougoslave avec trois chutes: Iuti, Sip (Gura-Vaii) et Gruia. Dans cette variante, on construirait d'abord l'usine amont de Iuti, où on améliorerait en mème temps les conditions difficiles de la navigation, qui n'ont pas pu être surmontées par la régularisation antérieure. Mais on n'utilise ainsi que $50 \%$ du potentiel des Portes de Fer el des conditions de navigation aux cataractes.

Une analyse économique a montré simplement que la meilleure solution est l'aménagement des Portes de Fer à une chute à Sip-Gura Vaii. Les travaux de reconnaissance géologique, entrepris en 1961 et 1962, ont montré que près de Sip-Gura Vaii, les deux rives et le lit du fleuve se trouvent dans le gneiss, les micaschistes, les amphibolites et les schistes quartzeux.

On a creusé 2400 trous de sondage sur les rives et les îles et environ 1800 dans le lit du fleuve; avec les sondages, on est allé jusqu'à la profondeur de $50 \mathrm{~m}$; sur les rives, on a creusé 8 galeries d'une longueur de $250 \mathrm{~m}$. Ces reconnaissances géologiques et les essais de pression, de cisaillement et de glissement ont confirmé les conclusions antérieures selon lesquelles le rocher, dans le profil de Sip-Gura Vaii, est favorable à la fondation d'un grand barrage.

De tous les profils pris en considération dans le défilé des Portes de Fer, celui de Sip-Gura Vaii est aussi le plus favorable du point de vue de l'organisation du chantier et des accès pour le transport des matériaux. On a choisi déjà les balastières et les carrières de pierre pour les agrégats concassés; le ciment sera transporté soit en bateaux directement sans déchargement sur d'autres moyens de transport, soit par les voies de chemin de fer ou les routes que l'on pourra sans difficultés amener jusqu'au chantier. On a examiné et exécuté de nombreux autres travaux d'investigation nécessaires à une bonne organisation du chantier.

Tout comme les conditions topographiques, géologiques et autres conditions techniques de la construction des ouvrages, l'aspect des circonstances hydrologiques est aussi très favorable. Au site choisi pour la construction de l'usine, le bassin versant du Danube possède une superficie d'environ $580000 \mathrm{~km}^{2}$ et un débit annuel de $175.10^{9} \mathrm{~m}^{3}$. La diversité des conditions climatiques dans le bas$\sin$ agit dans le sens de la diminution des diffé- 
rences entre les hautes et basses eaux. Ceci est surtout remarquable pour les débits mensuels moyens. On relève que le débit mensuel maximal, en avril, n'est que 1,8 fois le débit moyen de $5600 \mathrm{~m}^{3} / \mathrm{s}$, et 2,9 fois le débit minimal, celui du mois de septembre. De même, les débits calculés pour les différentes périodes de fréquence ne montrent pas d'aussi grandes différences, ainsi que c'est le cas pour les autres grands fleuves de l'Europe.

Dans le profil hydrométrique très stable de Orsova, les observations des hauteurs d'eau se font depuis plus de cent vingt ans, et loute une série des mesures du débit a été faite, de sorte qu'on peut se baser avec certitude sur cet élément des calculs économiques. En outre, un remarquable nombre de mesures effectuées stir le Danube el ses affluents en amont des Portes de Fer confirme les résultats des mesures et observations à Orsova.

Des différences relativement faibles des débits mensuels moyens se manifestent le plus favorablement dans l'uniformité de la production de l'énergie d'été et d'hiver qu'on peut réaliser aux Portes de Fer aménagées avec une chute de Sip-Gura Vaii. L'énergie d'été de $5,610^{\circ} \mathrm{k}$ Wh ne dépasse celle d'hiver que de $4 \%$. Enfin, l'usine de Sip-Gura Vaii possède également les caractéristiques d'une usine de pointe, chaque mètre d'abaissement du niveau de la retenue pendant l'exploitation représentant un volume de $140-150$ millions de $\mathrm{m}^{3}$ que l'on peut utiliser sur une chute moyenne de plus de $20 \mathrm{~m}$.

\section{Problèmes posés par la création du barrage}

Dans le défilé des Portes de Fer se trouvent plusieurs villages et petites villes qui vont ètre submergés. Le nombre d'habitants qu'il faudra déplacer et pour lesquels il faudra construire de nouvelles habitations, s'élève à environ 22000 .

La route le long de la rive gauche dans le défilé et une courte partie de la voie ferrée sur la mème rive, ainsi que les routes sur la rive droite du Danube qui ne traversent pas tout le défilé, seront également submergées. Il en sera de mème pour quelques ouvrages industriels et les quais également sur l'autre rive.

Les terrains agricoles sont ici de faible étendue et leur production est à peine suffisante pour nournir les villages isolés, dont les habitants vivent principalement de la pêche et de l'élevage du bétail. L'immersion de ces villages de faible altitude et de quelques autres ouvrages se montre inévitable dans toutes les variantes de l'aménagement des Portes de Fer.

En tenant compte de ces faits, ainsi que des conditions géologiques favorables au site du barrage et de la retenue, on voit que l'on ne rencontre pas d'exigences limitant le rehaussement du niveau du Danube. Reste à savoir si et dans quelle mesure le rehaussement de la crête du barrage, et, en relation avec cela, l'augmentation de la puissance et de la productibilité de l'usine, viendraient en conflit avec la nécessité de ne pas aggraver le régime du Danube et de ses affluents en amont ainsi que l'état d'importants terrains agricoles et des agglomérations situées le long du fleuve et de ses affluents. Il est évident que les deux pays ont un grand intérêt à ce que les conditions particulièrement favorables à la construction d'une puissante usine soient exploitées au maximum, c'est-à-dire à porter la chute utile jusqu'aux dernières limites possibles. La fixation de cette limite est précisément le problème le plus difficile des Portes de Fer. Presque toutes les limitations de la cote de la retenue proviennent du territoire yougoslave, car les effets de la retenue seront sensibles à une grande distance en amont, non seulement sur le Danube, mais aussi sur la Morava, la Tamis, la Theiss en leurs vallées.

\section{Fixation de la cote de retenue}

Trois questions fondamentales apparaissent dans ce problème : quelle sera la perturbation de l'équilibre dans le transport des sédiments, dont les dépôts seront auggmentés dans le lit du Danube, aussi bien que dans celui de ses affluents, notamment de la Morava? Dans quelle mesure et comment faudra-t-il renforcer les digues de défense 'qui ont bien résisté jusqu'ici aux crues de durée relativement courte? Et enfin, quelles seront les conséquences sur le régime des eaux souteraines dans les périmètres agricoles défendus ? Il est clair que seule une soution très ćlastique, même extrèmement élastique, de ces questions, devait être adoptée comme solution définitive; et c'est ce qui a été fait.

Puisque le défilé lui-mème ne posait pas de limites à l'augmentation de la retenue, sa cote à fixer devait être cherchée, non pas au barrage, mais à environ $130 \mathrm{~km}$ en amont de celui-ci, là où le Danube passe de la basse plaine, qu'il menace d'inondations, dans le défilé. A peu près à cet endroit, s'achèvent les digues de défense contre les inondations; là se trouve le confluent de la rivière Nera, qui forme une partie de la frontière entre la Roumanie et la Yougoslavie dans la plaine pannonienne; là est aussi le débouché du canal principal d'assèchement du système hydraulique Danube-Theiss-Danube.

Les études ont montré qu'en cet endroit (Bazias, en territoire roumain ou Banatska Palanka en territoire yougoslave) toutes les eaux avec des débits entre 2200 et $8500 \mathrm{~m}^{3} / \mathrm{s}$ peuvent être maintenues a la cote 69,50 , alors que pour des débits plus élevés, cela ne permet qu'un relèvement très faible du niveau. Cette solution comporte la condition de rehaussement maximal du niveau du Danube, mais on ne peut pas encore prévoir avec une précision suffisante l'effet dù au rehaussement sur les terrains protégés et sur la sédimentation dans le Danube et ses aflluents.

Pour cela, on prévoit dans la première période de l'exploitation que l'on ne doit pas limiter à la cote 68,00 le maintien des eaux à Banatska Palanka. Après une longue période d'observation des effets de cette hauteur de la retenue, on passera, dans le cas favorable, au relèvement du niveau à la cote définitive de 69,50 , au point caractéristique de la ligne de remous.

Sur les bases d'une telle solution, on a suggéré aux constructeurs de prévoir des parties mobiles au barrage Sip-Gura Vaii, de façon à pouvoir ultérieurement réaliser l'exhaussement de la retenue sans constructions complémenlaires. Ceci permet, dans la première phase d'exploitation du système, de maintenir les niveaux supérieurs à la cote 68,00 lorsque les eaux du Danube et ses principaux affuents coulent limpides ou peu chargées de sédiments. Alors on étudierait sur place l'effet des hautes eaux de durée arbitraire sur la stabilité des 


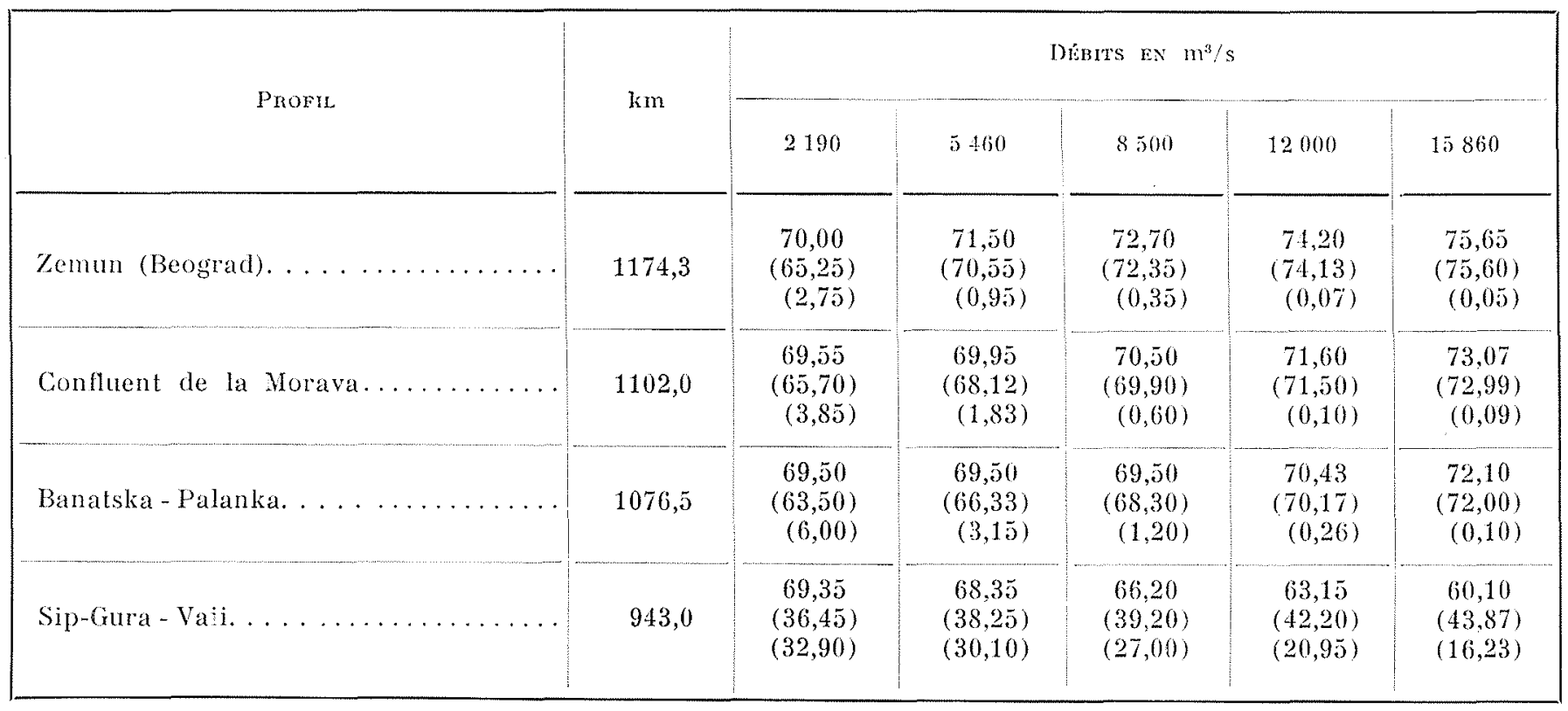

digues de défense et l'état des eaux souterraines, ainsi que l'effet sur les dépôts de sédiments dans les lits du Danube et de ses affluents.

\section{Effet du remous}

Le tableau ci-dessus indique les cotes des eaux surélevées et des eaux non surélevées, des plus petites jusqu'aux plus grandes pour quelques points choisis de la ligne de remous, présentant un intérêt particulier. Le calcul du remous est fait sur la base des données morphologiques et hydrologiques récentes.

Les niveaux surélevés sont calculés à quelques profils caractéristiques. A Banatska Palanka le niveau est maintenu à la cote 69,50 pour les débits entre 2200 et $8500 \mathrm{~m}^{3} / \mathrm{s}$.

Entre parenthèses sont les cotes des eaux non surélevées et les hauteurs de surélévation aux profils correspondants.

La surélévation de $2,75 \mathrm{~m}$ des basses eaux à Beograd rendra possible l'accès de la capitale de la Yougoslavie aux bateaux montants d'une capacité de portée de 500 t. L'exhaussement notable des basses eaux à Banatska Palanka fait que la ligne de remous s'étend en amont du confluent de la Theiss et presque au confluent de la Drave (km 1380 ). Le drainage du Banat méridional sera ainsi rendu plus difficile.

Le relèvement des niveaux aux débits maximaux en amont de Banatska Palanka est faible et n'aura pas d'influence sur une surélévation de couronne des digues de protection.

Les plus grandes difficultés proviemnent des eaux moyennes et des caux qui, à l'état naturel, remplissaient le lit où se déversaient pendant de courtes périodes jusqu'au pied des digues de défense. Comme conséquence du long séjour des eaux au niveau relevé apparait le besoin de la reconstruction des digues, qui ne peuvent pas résister à une longue action de l'eau. Une autre conséquence est la nécessité de reconstruire les stations de pompage et une augmentation des dépenses

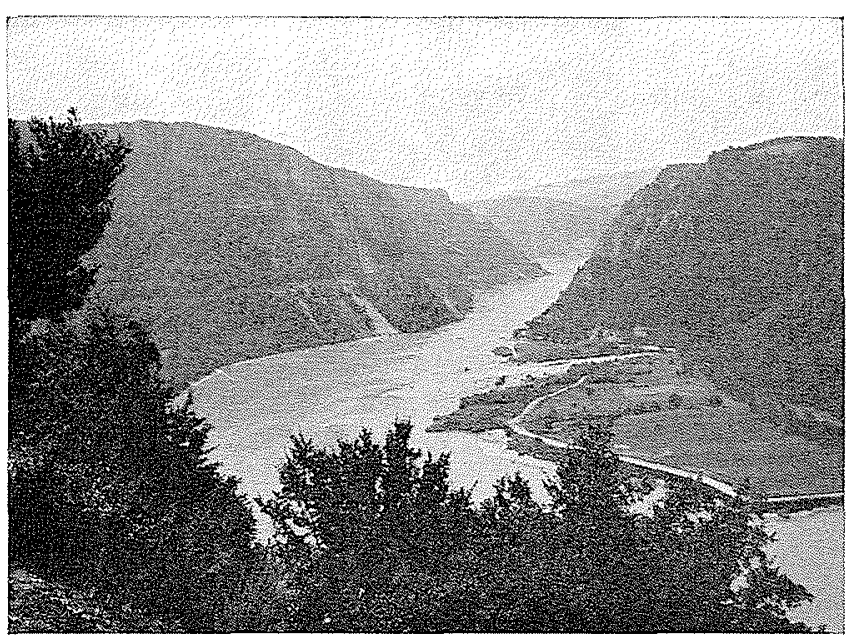

Chenal de sip.

Die kanalisierte Donaustrecke bei Sip.

d'énergie pour maintenir le niveau des eaux souterraines à l'état naturel. Finalement, troisième conséquence, la plus lourde et de la plus grande portée, est le changement nuisible du régime du transport des sédiments dans le lit du Danube jusqu'au confluent de la Theiss et qui se manifestera, avec des suites défavorables sur la sédimentation dans le lit de la Morava et sur l'aménagement de sa large et fertile vallée. Le problème de l'aménagement de la vallée et du bassin versant de la Morava, ainsi que de la régularisation de son lit, en cours d'exécution, est devenu un des plus compliqués dans le cadre de l'utilisation maximale du potentiel énergétique des Portes de Fer.

Jusqu'au confluent de la Morava, le Danube transporte environ $35.10^{\circ} t$ de matériaux en suspension et environ 2,5.10 $t$ de matériaux charriés. La composition granulométrique du sable est uniforme et bien définie par $\mathrm{d}_{50}=0,245 \mathrm{~mm}$ et $\mathrm{d}_{90}=0,360 \mathrm{~mm}$. La Morava apporte au total en- 
viron $13,5.10^{6} \mathrm{t}$ et la part des matériaux charriés est estimée à $3-10 \%$ d'après les mesures effectuées au cours de quatre années avec un bathymètre du type Korolyi et les calculs basés sur des formules empiriques et sur des analyses très détaillées des processus d'érosion dans son bassin versant. La composition du débit solide charrié est $\mathrm{d}_{50}=6 \mathrm{~mm}$ et $\mathrm{d}_{90}=20 \mathrm{~mm}$. En 1955 , la Morava a transporté $13570000 \mathrm{t}$, et en $1961,3910000 \mathrm{t}$ de sédiments en suspension et charriés.

D’après une analyse soignée des coüncidences des hauteurs d'eau dans le Danube et des transports de sédiments de la Morava, il a été calculé, qu'après l'exécution des travaux aux Portes de Fer, 1,5 à 2 millions de $\mathrm{m}^{3}$ de son transport annuel (d'environ 10 millions de $\mathrm{m}^{3}$ ), se déposeront dans son lit. Le remous du Danube s'étendra à $20 \mathrm{~km}$ du cours régularisé de la Morava. Pour une largeur du lit de la Morava d'environ $125 \mathrm{~m}$, il faut s'attendre à un relèvement de 0,6 à $0,8 \mathrm{~m}$ du fond $\mathrm{du}$ lit sur cette longueur. Le reste sera apporté au Danube, où il se déposera au moins $300-400000 \mathrm{~m}^{3}$ par an.

\section{Description des ouvrages}

Au milieu du lit du fleuve sera placé le barrage, avec 14 pertuis déversoirs d'une largeur de $25 \mathrm{~m}$. Ils sont séparés par 13 piles d'une épaisseur de $7 \mathrm{~m}$, ce qui donne au barrage une largeur totale de $441 \mathrm{~m}$. Le seuil déversoir est à la cote 55,2. Il est surmonté par une vanne mobile à deux corps, qui retient tous les débits inférieurs à $8500 \mathrm{~m}^{3} / \mathrm{s}$ à la cote 69,50 et les débits supérieurs à des cotes plus basses. L'épaisseur de la veine déversante pour le débit maximal est de $10,6 \mathrm{~m}$. La veine se brise sur un très court radier en forme de saut de ski. Le type de radier est étudié aux laboratoires d'hydraulique à Bucarest et à Belgrade.

Deux salles de machines identiques sont logées de part et d'autre du barrage; chacune a $200 \mathrm{~m}$ de long. Six turbines Kaplan y seront installées, c'est-à-dire au total 12 turbines pour un débit $Q_{\text {inst. }}$ $=8500 \mathrm{~m}^{3} / \mathrm{s}$ et une chute d'environ $26 \mathrm{~m}$. La chute maximale aux basses eaux du Danube sera de $34 \mathrm{~m}$. La puissance totale installée des groupes générateur's est de $2100 \mathrm{MW}$.

Entre le bâtiment des machines et la rive passent, des deux côtés du fleuve, les écluses de navigation. On a fait plusieurs variantes, dont l'une est montrée sur la photographie de la maquette. L'écluse y est à une chute et, comme telle, elle doit soutenir la comparaison avec l'écluse à deux chutes, plus chère mais propice à l'exploitation. Les écluses à deux chutes ont deux sas d'une longueur de $300 \mathrm{~m}$ chacun et une largeur de $34 \mathrm{~m}$. Du côté amont, le seuil est à la cote 58,50 et présente deux entailles dans son corps, une pour la ranne de commande et l'autre en réserve. Le fond du sas amont est à la cote 47,50 ; celui du sas aval, à 30,50 . Le niveau le plus bas du sas inférieur est à la cote 35,0 et le plus haut à la cote 52,0 .

Les systèmes de remplissage des sas de l'écluse à deux chutes sont à l'étude, en coordination dans les laboratoires hydrauliques à Belgrade et à Bucarest. On prévoit un temps de remplissage du sas amont de 9 à $10 \mathrm{mn}$ et du sas aval en $11 \mathrm{mn}$.

Entre les écluses de navigation et les rives hautes, sont placés des barrages non déversants en béton.

\section{Approfondissement du lit en aval du barrage}

La position du barrage au milieu du profil fluvial et la disposition symétrique des bâtiments des machines, des turbines et des écluses de navigation dans une partie rectiligne du fleuve assurent les mêmes conditions d'exploitation aux installations yougoslaves et roumaines. En aval du barrage, les conditions ne sont pas égales sur les deux côtés

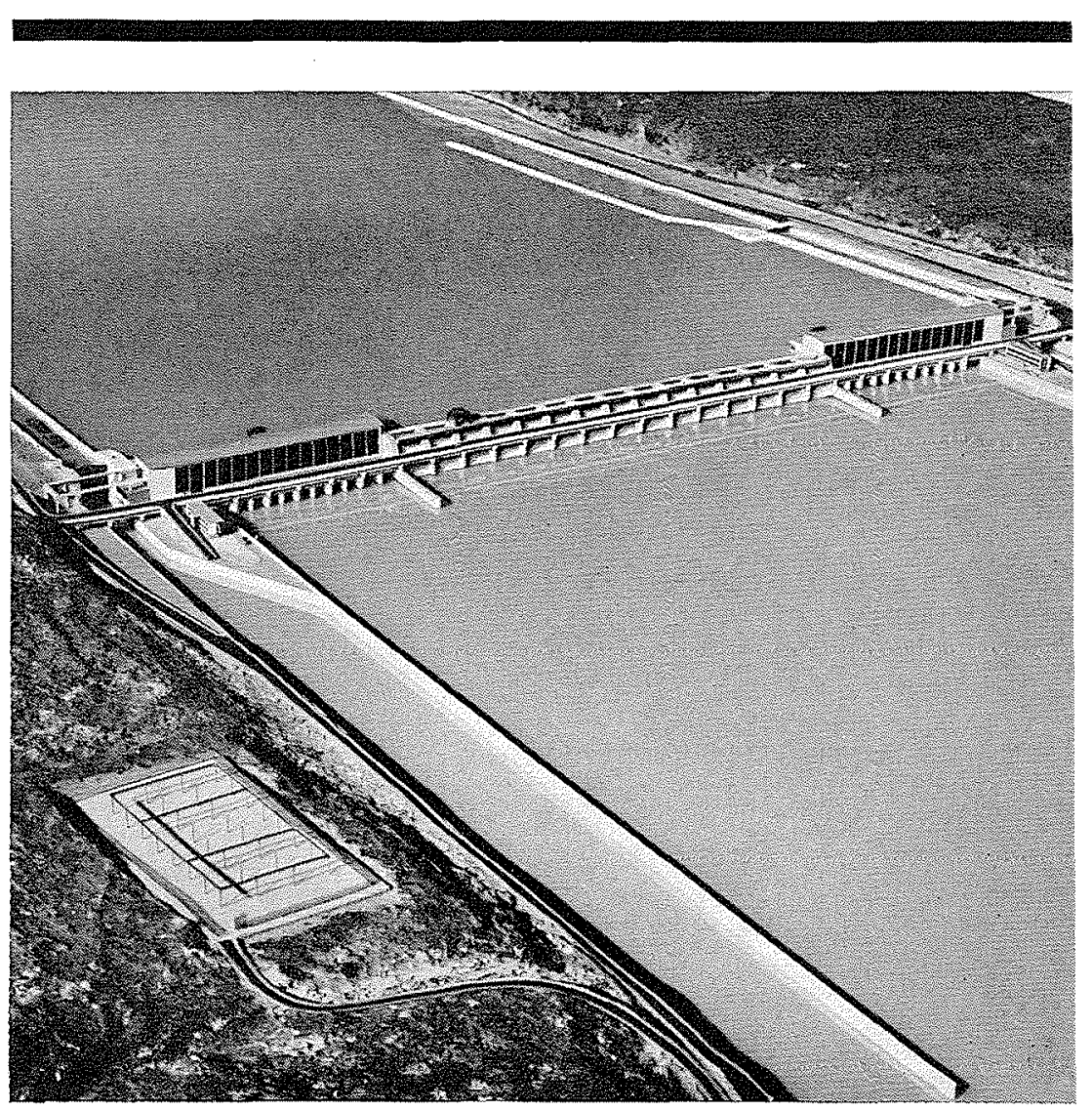

Maquette du barrage

et de la centrale «Djerdap».

Modell der Staumauer

und des Kraftwerks $\ll$ Djerdap $»$.

du fleuve, et il faudra les égaliser au prix de travaux importants.

A une courte distance en aval du barrage, sur le côté droit du lit, on rencontre cinq iles qui empêchent l'accès à l'écluse de navigation et rejettent la majeure partie du courant vers la rive gauche où l'accès à l'écluse est assuré.

Pour égaliser les conditions d'écoulement, le projet prévoit le creusement le long de la rive droite d'un chenal large de $250+95 \mathrm{~m}$. Avec les matériaux excavés, on formera une nouvelle île au milieu du lit, de sorte que, sur le côté gauche du fleuve, on réalisera la même largeur du lit de $250+95 \mathrm{~m}$. Le fond du chenal sera à la cote moyenne 29,0 .

Sur une longueur d'environ $4 \mathrm{~km}$ en aval du barrage, la pente du fleuve est de $0,44 \%$. Cela permet, par un approfondissement du lit, d'augmenter la chute de l'usine de $1,2 \mathrm{~m}$ et représente un gain d'environ $340.10^{6} \mathrm{kWh}$ par an; cela méritait d'être pris en considération. 


\section{Les travaux principaux}

Les travaux principaux ont l'ampleur suivante; déblais d'alluvions et de roches : 18 millions de $\mathrm{m}^{3}$, béton et béton armé 21,4 millions de $\mathrm{m}^{3}$; acier $34000 \mathrm{t}$; plus de $30000 \mathrm{~m}$ de trous d'injections. Le cube des déblais est fortement influencé par l'approfondissement du lit en aval du barrage, et le cube de béton, par les deux écluses de navigation à deux chutes. A ces quantités, il faut ajouter encore 15-20 $000 \mathrm{t}$ d'acier pour les batardeaux. L'ensemble du prix de tous ces travaux, des machines et de l'équipement approche de la somme de 400 millions de dollars.

\section{L'exécution des travaux}

Il y a trois exigences à satisfaire : les conditions de la navigation pendant la durée des travaux ne doivent pas être moins favorables que dans le canal de Sip, où les vitesses ne dépassent pas $5 \mathrm{~m} / \mathrm{s}$; le chantier doit être protégé contre les débits de $15000 \mathrm{~m}^{3} / \mathrm{s}$ (période de retour : soixante-dix ans); il faut prévoir une défense sûre contre les glaces.

La première condition sera satisfaite par la largeur de la passe navigable entre les batardeaux de la fouille et par l'établissement de la traction auxiliaire, non plus à l'aide de locomotives comme dans le canal de Sip ou du toueur dans le canal de Iuti, mis avec des remorqueurs de $2500 \mathrm{ch}$.

La deuxième et la troisième conditions demandent que le bord supérieur du batardeau soit à la cote 46,0 (et à la cote 53,0 dans la III $^{\circ}$ phase de construction), que son corps possède une imperméabilité suffisante et qu'il résiste aux pressions de la charge hydraulique et aux actions des glaces. Dans les parties profondes du lit, on prévoit la construction des batardeaux en cellules circulaires de 18 à $20 \mathrm{~m}$ de diamètre, formées de palplanches en acier remplies de matériaux dragués. Près des rives, où les profondeurs et les vitesses sont moindres, les batardeaux seront faits en pierres avec écran métallique.

On prévoit trois phases de construction sous la protection des batardeaux et une phase après leur enlèvement et la mise en service des premiers groupes de générateurs installés.

Dans la première phase, l'enceinte comprendra les écluses de navigation et les bâtiments des machines des deux côtés du lit, avec, au côté droit, également trois travées du barrage déversoir. II reste au milieu la passe navigable, d'une largeur de $300 \mathrm{~m}$. La longueur des batardeaux est de $1400 \mathrm{~m}$ avec cellules circulaires et environ $1100 \mathrm{~m}$ de digues avec écran.

Dans cette phase et dans les suivantes, le barrage déversoir sera construit à des hauteurs différentes, alors que les piles et les ponts qu'elles portent seront construits entièrement. La navigation dans la première et dans la seconde étapes s'effectuera dans le lit du fleuve et, avec le commencement de la troisième étape, par le sas inférieur achevé et le sas supérieur inachevé de l'écluse de navigation sur la rive roumaine. Au cours de la quatrième étape, seront mis en service deux groupes générateurs dans chaque bâtiment des machines. Dans cette étape, on achèvera l'approfondissement du lit en aval du barrage, l'enlèvement de tous les batardeaux, alors que les parties déversantes du barrage et les écluses seront terminées sous la protection des vannes-batardeaux.

Les projets de tous les ouvrages et installations ainsi que les plans d'exécution ont été élaborés en détail au bureau «Energoprojekt » à Belgrade et à l'Institutul de studii si proiectari energetici (I.S.P.E.) à Bucarest. Les recherches sur modèles hydrauliques se font à Interprinderes de cercatari si studii de teren hidroenergetica (I.C.S.E.) à Bucarest et au Laboratoire Avala de l'Institut des eaux à Belgrade.

Les travaux de construction doivent commencer en 1964 et c'est en 1971 que l'usine hydroélectrique «Djerdap » doit entrer en service.

\section{Bibliographie}

[1] Petrovic (N.) : Deux expeditions hydrographiques dans le secteur du Danube nommé Portes de Fer depuis 130 ans. (En serbo-croate). Inst. des Eaux, 1960. L'auteur évoque les relevés des Portes de Fer, faits par le colonel Jankovic de 1830 à 1832

[2] Dukic (D.) : Nos rivières. (En serbe) Beograd, 1952.

[3] Dragovic (R.): Plovidba u Djerdapu (La navigation aux Portes de Fer), 205 pages, Beograd, 1960.

4.] Urosevic (R.) et Mroosevic (M.) : Osnovne koncepeije hidroenergetskog i plovidbenog sistema HE Djerdap. (En serbe). Izgradnja, 1962, No 8 , Beograd.

\section{Zusammenfassung \\ Die Probleme des Eisernen Tors Von B. Knezevic *}

Unter dem Eisernen Tor versteht man den Teil der Donau, der an der rumänisch-jugoslawischen Grenze liegt. Er ist $117 \mathrm{~km}$ lang (km 931-1048) und umfasst fünf Stromschnellen-Abschnitte, deren bedeutendster der von Sip-Gura Vaii mit einem Gefälle von $7,6 \mathrm{~m}$ ist. Das Gesamtgefälle dieser Abschnitte beträgt $20,8 \mathrm{~m}$. Es handelt sich um einen $50 \mathrm{~m}$ tiefen Durchbruch, in dem sich die Donau auf $150 \mathrm{~m}$ verengt. Die Regulierung dieses Abschnitts, die 1834 aus Gründen der Schiffahrt begonnen wurde und $1900 \mathrm{zum}$ grossen Teil abgeschlossen war, umfasste die Ausbildung von Kanälen durch die Stromschnellen - insgesamt über eine Länge von $15,5 \mathrm{~km}$ - und den Bau von Deichen. Veber eine Million Kubikmeter Fels wurden ausgehoben. Es blieben jedoch weiterhin gewisse Schwierigkeiten bestehen : in den engen Durchgängen und den ausgegrabenen Kanälen kann die Schiffahrt nur einbahnig erfolgen, nachts ist sie untersagt; bei Niederwasser können die Schiffe nicht voll beladen werden.

Erst durch den Bau der in Höhe von Sip-Gura Vaii (km 943) vorgesehenen Talsperre wird das Schiffahrtsproblem im Gebiet des Eisernen Tors endgültig gelöst werden. Durch diese Sperre, deren Bau im Frühjahr 1964

Früher Professor an der Fakultät für Bauingenieurwesen Belgrad (Ungarn). 


\section{B. KNEZEVIC}

anlaufen soll, wird es möglich, $80 \%$ des hydraulischen Potentials dieses Gebiets, das jährlich insgesamt $12,5.10^{9} \mathrm{kWh}$ beträgt, nutzbar zu machen. Die Gesamtleistung des Kraftwerks beträgt $2100 \mathrm{MW} ; Q_{\text {inst. }}=8500 \mathrm{~m}^{3} / \mathrm{s}$. Ein beträchtlicher Teil des Artikels ist diesem Bauwerk gewidmet.

Das Hauptproblem dieses Bauwerks bildete die Bestimmung der Stauhöhe. Die Begrenzung des Niveaus lag nicht im Durchbruch selbst, sondern in der stromaufwärts liegenden Niederung, da der Rückstau bis tief in die Niederung hinein reichen und nicht nur die Ufergelände der Donau, sondern auch die der Morava, der Sau und der Theiss beeinflussen wird. Es hat sich gezeigt, dass eine Zunahme der Hochwasserführung in der Niederung nicht zugelassen werden kann, denn eine solche kann nur bei Niederwasser und Mittelwasser aufgenommen werden. Eine komplexe Analyse fûhrte zur Wahl eines Profils am Eintritt in den Durchbruch (Profil von Bačka Palanka), wo eine Kote festgesetzt wurde; während der Nutzung des Staus müssen seine Spiegelhöhen ungefähr bei dieser Kote liegen. Die endgültige Kote bei diesem Profil wurde auf ungefähr $69,5 \mathrm{~m}$ festgesctzt, die Koten in Höhe der Sperre auf 63,0 bei Hochwasser und auf 69,35 bei Niederwasser. In einer ersten Phase wird die Kote 68,0 gewählt (beim Profil von Bačka Palanka). Trotz eingehender Vntersuchungen musste bei der Festsetzmng dieser Höchstkote sehr vorsichtig vorgegangen werden. Diese Vorsicht ist bedingt durch die komplexen Wirkungen des Rückstaus :

- auf die zum Schutz gegen Ueberschwemmung errichteten Deiche, die verstärkt werden müssen, da sie nicht so ausgeführt sind, dass sie auf lange Sicht hinaus der Einwirkung des Wassers widerstehen können;

- das Grundwasserregime erfordert den Bau eines neuen Entwässerungssystems;

- das Regime der Geschiebefracht, vor allem die Ablagerung des Geschiebematerials am Grunde des Flusses, führen zu Spiegelerhöhungen.

Eine volle Verwendung des Bauwerks (Kote 69,5) ist erst nach einer ersten Erfahrung durch die Nutzung des Staus bei Kote 68,0 vorgesehen.

Die hydraulischen Untersuchungen wurden in rumänisch-jugoslawischer Zusammenarbeit mit Hilfe von Versuchsmodellen, Elektronenrechnern usw. ausgeführt.

Hier einige Daten des Bauwerks :

Die Gevichstmauer umfasst 14 Wehröthungen, von denen jede $25 \mathrm{~m}$ breit ist und mit einem $14 \mathrm{~m}$ hohen beweglichen Schütz ausgerüstet ist (in vollendetem Zustand). Die auf beiden Seiten der Staumauer vorhandenen Maschinenräume enthalten je 6 Kaplanturbinen. Fallhöne : 11 bis $34 \mathrm{~m}, 26 \mathrm{~m}$ im Mittel.

Zwei Schleusen $(300 \times 34 \mathrm{~m})$. Durch Vertiefung des Betts unterhalb der Staumauer wird die Fallhöhe um $1,2 \mathrm{~m}$ vergrössert (was gleichzeitig auch den Zugang zu den Schleusen erleichtert).

Ueber den Umfang dieser Arbeiten kann gesagt werden, dass ungefähr 18 Millionen Kubikmeter Ablagerungen und Fels ausgehoben werden und 21,4 Millionen Kubikmeter Beton und Stahlbeton sowie 3400 Tonnen Stahl zur Verwendung kommen.

Die Arbeiten sollen bis 1971 abgeschlossen sein.

Entre le moment où les deux articles précédents ont été rédigés par le Professeur Bogič KNEZ̈EVIĆ et la partution de notre numéro, nous avons appris anec peine son décés survenule 12 mai 1963.

Ancien rectenr de l'Ecole Polytechnique Supérieure de Belgrade, le Professeur Bogič KNEZ̈EVIC̈ avait dirigé l'INSTITUT DES EAUX «JAROSLAV CERNI》.

Fondateur et Président de l'Association Yougoslave de Recherches Hydrauliques, il fut aussi Lauréat du Conseil National de Recherches Scientifiques. 